\title{
INVESTIGAÇÃO DE PARÂMETOS REACIONAIS DA SÍNTESE DE FISCHER-TROPSCH EM UMA UNIDADE DE BANCADA UTILIZANDO CATALISADOR DE Co-Pt $/ \gamma-\mathrm{Al}_{2} \mathrm{O}_{3}$
}

\author{
V. VITAL ${ }^{1}$, M. BEZERRA ${ }^{1}$, L. MARANHÃO ${ }^{1}$, N.L. FILHO $^{1}$, L. C. ALMEIDA $^{1}$ \\ ${ }^{1}$ Universidade Federal de Pernambuco, Departamento de Engenharia Química \\ E-mail para contato: vitalqi@yahoo.com.br
}

\begin{abstract}
RESUMO - Como principal reação da tecnologia Gas to Liquids (GTL), a síntese de Fischer-Tropsch surgi como alternativa expoente para enriquecimento da cadeia do gás natural. Utilizando uma unidade de bancada que possibilita a reação e a análise dos produtos formados. Foram realizadas reações a pressão constante de 2,0 $\mathrm{MPa}$ e relação molar de gás de síntese $\mathrm{H}_{2}: \mathrm{CO}=2: 1$, com um catalisador de $20 \% \mathrm{Co}-0,5 \% \mathrm{Pt} / \gamma-\mathrm{Al}_{2} \mathrm{O}_{3}$, observando a influência da temperatura $\left(185\right.$ a $\left.230^{\circ} \mathrm{C}\right)$ e velocidade espacial $(0,4$ a 3,0 mols gás.gcat $\left.{ }^{-1} \cdot h^{-1}\right)$. Os resultados obtidos mostraram conversões de $\mathrm{CO}$ na faixa de 3,7 a 99,8 \% e seletividades a $\mathrm{CH}_{4}$ e $\mathrm{C}_{5+}$ nas faixas de 3,7 a $55 \%$ e 38 a 95,1\%, respectivamente. Ficou evidenciado, nas condições estudadas, que conversões acima de 80\% acarretaram um aumento da seletividade a $\mathrm{CH}_{4}$ de forma exponencial. Por outro lado, altos valores de velocidade espacial tem uma contribuição mais significativa na seletividade do $\mathrm{CH}_{4}$, enquanto que o parâmetro temperatura tem maior influencia sobre a conversão de CO. Para uma mesma iso-conversão, o rendimento a hidrocarbonetos líquidos, menor \% $\mathrm{CH}_{4}$, é favorecido por condições temperatura e velocidades mais elevadas, onde os efeitos de diluição diminuem o comportamento exotérmico da reação.
\end{abstract}

\section{INTRODUÇÃO}

Segundo De la Osa et al. (2012), a elevada flexibilidade na produção de uma gama de produtos e seu apelo ambiental, tornam a síntese de Fischer-Tropsch (SFT) a mais promissora reação para produção de hidrocarbonetos e compostos oxigenados de alto valor agregado na tecnologia Gas to Liquids (GTL). Esta flexibilidade está condicionada a vários fatores, tais como a formulação do catalisador final, bem como uma variedade de parâmetros operacionais, e portanto, tornando-se imprescindível a otimização dos parâmetros do processo para obtenção de produtos petroquímicos, de forma eficiente, com elevado valor comercial.

Segundo Wang et al. (2013), os catalisadores a base de Co são os mais utilizados, pois possuem elevadas estabilidade, atividade e alta seletividade a hidrocarbonetos $\mathrm{C}_{5_{+}}$, além da baixa tendência para a reação Water Gas Shift (WGS). Para melhorar algumas propriedades dos catalisadores, muitas vezes, faz-se necessário o uso de promotores, com a Pt sendo utilizada em vários trabalhos realizados: Jacobs et al. (2002); Jermwongratanachai et al. (2013) e Wang et al. (2013). 


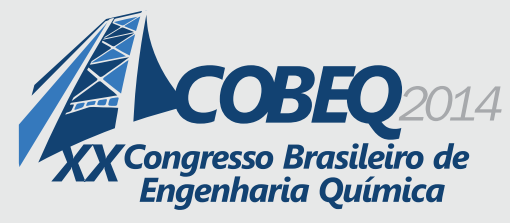

19 a 22 de outubro de 2014
Florianópolis/SC

Apesar da importância dos catalisadores, mudanças nas condições operacionais podem influenciar nos indicadores da reação, tais como seletividades e conversão. Segundo Yang et al. (2014), um aumento da temperatura permite diferentes produtos formados na reação da SFT, pois pode ocorrer uma preferência por reações secundárias. Outro parâmetro operacional que pode influenciar na seletividade da SFT é a velocidade espacial, segundo Everson et al. (1978) e Ma et al. (2012), eles relataram que há uma relação direta da conversão de $\mathrm{CO}$ e a formação de $\mathrm{CH}_{4}$. Portanto, como objetivo principal, este trabalho propõe observar a influência da temperatura e da velocidade espacial sobre os produtos da reação de FT.

\section{PROCEDIMENTO EXPERIMENTAL}

\subsection{Preparação do Catalisador}

A $\gamma-\mathrm{Al}_{2} \mathrm{O}_{3}$ (Sasol Catalox-150) foi utilizado como suporte catalítico. A mesma foi calcinada por $5 \mathrm{~h}$ a $550^{\circ} \mathrm{C}$ numa mufla. Os precursores utilizados para a impregnação dos metais $\mathrm{Co}$ e $\mathrm{Pt}$, foram o sal nitrato de cobalto hexahidratado $\left[\mathrm{Co}\left(\mathrm{NO}_{3}\right)_{2} \cdot 6 \mathrm{H}_{2} \mathrm{O}\right]$ e uma solução de hidróxido tetramina platina (II) $\left[\mathrm{Pt}\left(\mathrm{NH}_{3}\right)_{4}(\mathrm{OH})_{2}\right]$. A fase ativa, metal Co, com teor de $20 \% \mathrm{p} / \mathrm{p}$, foi impregnada utilizando a técnica de impregnação umidade incipiente em co-precipitação com a platina, com teor de $0,5 \% \mathrm{p} / \mathrm{p}$. A impregnação foi realizada de forma sucessiva em três etapas de mesmo volume de poros do suporte. A cada impregnação o catalisador foi secado a $120^{\circ} \mathrm{C}$ durante $12 \mathrm{~h}$. Após a última impregnação, a amostra foi calcinadas a $350^{\circ} \mathrm{C}$ por $6 \mathrm{~h}$ usando-se uma rampa de aquecimento de $1^{\circ} \mathrm{C} \cdot \mathrm{min}^{-1}$. O catalisador, $\mathrm{Co}-\mathrm{Pt} / \gamma-\mathrm{Al}_{2} \mathrm{O}_{3}$, foi caracterizado via técnicas de quimissorção de $\mathrm{H} 2, \mathrm{AAS}, \mathrm{TPR}, \mathrm{DRX}$, BET e IFTR. As caracterizações do catalisador não serão mostradas neste trabalho.

\subsection{Testes Catalíticos}

A SFT foi realizada em uma unidade escala de bancada. O sistema está composto por um reator tubular (leito fixo) de aço inoxidável (d.i. $0,8 \mathrm{~cm}$ ), um forno automático (ThermoScientific, Lindberg blue M) e um cromatógrafo a gás online (Agilent Technologies, 7890A GC System). O gás de síntese utilizado consiste numa mistura gasosa com proporção molar de $\mathrm{H}_{2}: \mathrm{CO}$ de $2: 1$, composta por $3 \% \mathrm{~N}_{2}$, $10 \% \mathrm{CO}, 20 \% \mathrm{H}_{2}$ e $\mathrm{He} 67 \%$.

O catalisador utilizado $\left(20 \% \mathrm{Co}-0,5 \% \mathrm{Pt} / \gamma-\mathrm{Al}_{2} \mathrm{O}_{3}\right)$ foi diluído com carbeto de silício (SiC), numa proporção mássica de 1:5. A redução catalítica foi realizada in situ com vazão de $\mathrm{H}_{2}$ de $120 \mathrm{~mL}$.min ${ }^{1}$. gcat ${ }^{-1}$, pressão de 1 bar, a $360^{\circ} \mathrm{C}$ com duração de $10 \mathrm{~h}$ e taxa de aquecimento de $1^{\circ} \mathrm{C}$. $\min ^{-1}$. Ao término da redução, o reator foi resfriado para $170^{\circ} \mathrm{C}$. O sistema foi submetido a pressão de $2,0 \mathrm{MPa}$, com uma mistura gasosa contendo o gás de síntese, dando início as condições reacionais.

Posteriormente, para cada sequência de reações experimentais, a vazão de gás de síntese foi fixada e variou-se a temperatura (T) de reação, iniciando-a em $230^{\circ} \mathrm{C} \rightarrow 215^{\circ} \mathrm{C} \rightarrow 200^{\circ} \mathrm{C} \rightarrow 185^{\circ} \mathrm{C}$ (utilizando-se uma taxa de $1^{\circ} \mathrm{C} \cdot \mathrm{min}^{-1}$ ). Cada sequência experimental teve duração de aproximadamente $120 \mathrm{~h}$, sendo considerado para cada condição de temperatura cerca de $30 \mathrm{~h}$ de reação (tempo observado para o sistema alcançar o estado estacionário). Os resultados foram uma média de 5 pontos no estado estacionário, cerca de $10 \mathrm{~h}$ de reação. Um condensador a $90^{\circ} \mathrm{C}$ foi 
utilizado para remoção de hidrocarbonetos pesados e produtos líquidos entre o reator e o cromatógrafo a gás. Os gases produzidos $\mathrm{H}_{2}, \mathrm{CH}_{4}, \mathrm{CO}_{2}$, padrão interno $\mathrm{N}_{2}$, hidrocarbonetos leves (C2-C4) e a conversão de CO, foram quantificados por meio de um (TCD). Finalizada as $120 \mathrm{~h}$ de experimento, o reator foi descarregado e lavado com n-hexano a $400^{\circ} \mathrm{C}$ por $3 \mathrm{~h}$ com aquecimento de $10^{\circ} \mathrm{C} \cdot \mathrm{min}^{-1}$.

Foram realizadas sequências de reações experimentais para as velocidades espaciais $\left(\mathrm{v}_{\mathrm{e}}\right)$ de 0,4 , $0,8,1,5$ e 3,0 mols gás.gcat ${ }^{-1} \cdot \mathrm{h}^{-1}$, mantendo-se a mesma vazão da mistura contendo o gás de síntese e variando-se a massa de catalisador.

\section{RESULTADOS E DISCUSSÃO}

\subsection{Tempo de reação}

A conversão de $\mathrm{CO}$ em conjunto com a seletividade aos produtos $\mathrm{CH}_{4}, \mathrm{CO}_{2}$ e $\mathrm{C}_{5+}$, são indicadores de eficiência na SFT. A Figura 1 apresenta os resultados obtidos ao longo do tempo para o catalisador $20 \% \mathrm{Co}-0,5 \% \mathrm{Pt} / \gamma-\mathrm{Al}_{2} \mathrm{O}_{3}$.
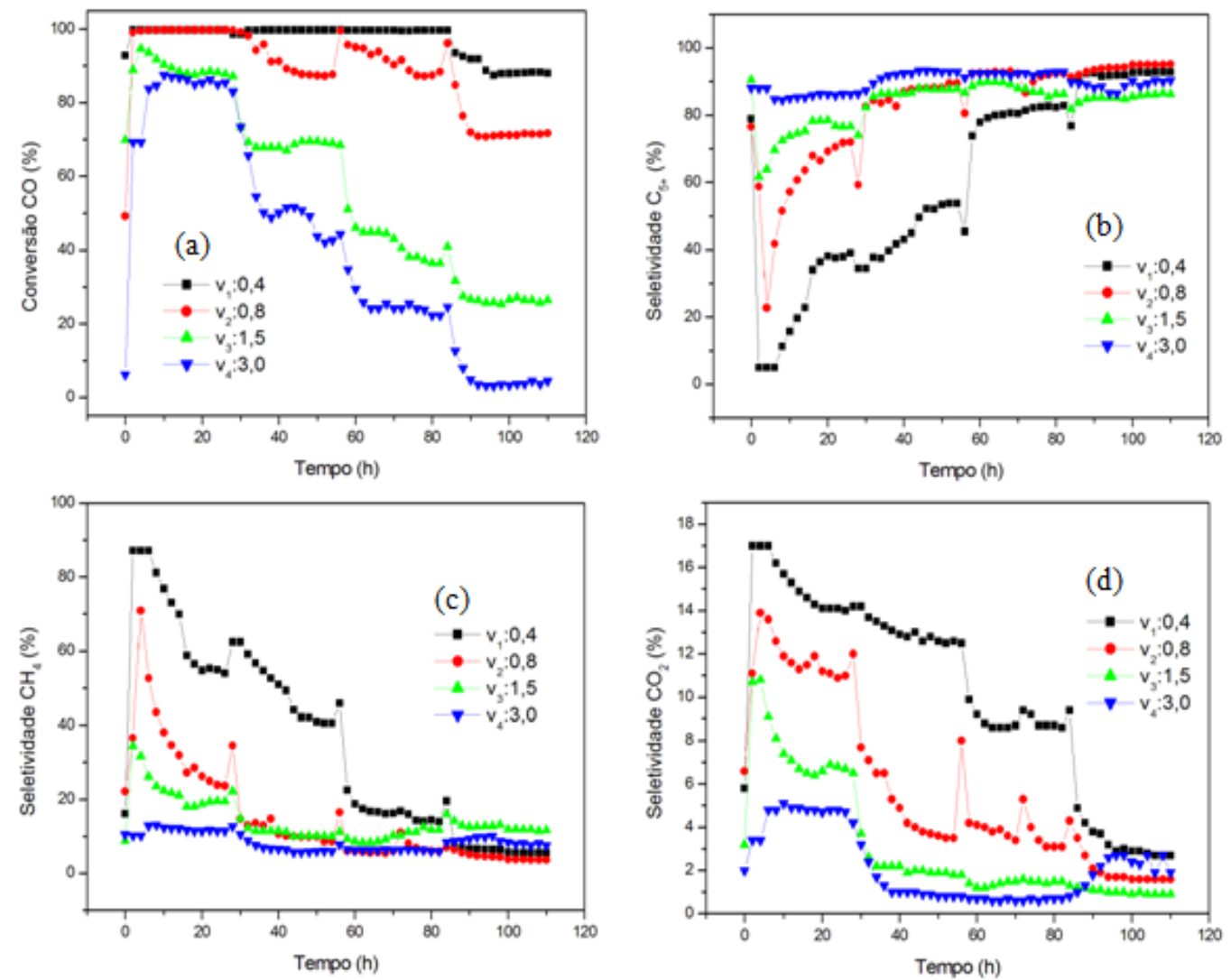

Figura 1 - Variação dos indicadores da SFT (a) conversão de CO, e seletividades a (b) $\mathrm{C}_{5+}$, (c) $\mathrm{CH}_{4} \mathrm{e}(\mathrm{d}) \mathrm{CO}_{2}$, sobre as variáveis do processo temperatura: $\mathrm{T}=230^{\circ} \mathrm{C}(0$ a $28 \mathrm{~h}), \mathrm{T}=215^{\circ} \mathrm{C}(28 \mathrm{a}$

$56 \mathrm{~h}), \mathrm{T}=200^{\circ}(56-84 \mathrm{~h})$ e $\mathrm{T}=185^{\circ} \mathrm{C}(84-112 \mathrm{~h})$; e velocidades espaciais: $\mathrm{v}_{1}, \mathrm{v}_{2}, \mathrm{v}_{3} \mathrm{ev}_{4}$. 


\section{9 a 22 de outubro de 2014 \\ Florianópolis/SC}

Na figura 1(a), de modo geral, observou-se um decréscimo na conversão de CO com a diminuição da temperatura e o aumento da velocidade espacial, exceto as primeiras $28 \mathrm{~h}$ de maior temperatura e menor velocidade espacial, onde a conversão de $\mathrm{CO}$ se manteve próxima a $99 \% . \mathrm{Na}$ figura 1(b), constatou-se um aumento na seletividade a $\mathrm{C}_{5+}$ com o aumento da velocidade espacial nas primeiras 58 h.Sugerindo que a velocidade espacial tem maior influência na obtenção de HC líquidos. A partir de $60 \mathrm{~h}$, a seletividade a $\mathrm{C}_{5+}$ converge para valores próximos a $85 \%$, independente da velocidade espacial.Na Figura 1(c), observou-se uma diminuição na seletividade a $\mathrm{CH}_{4}$ com o aumento da velocidade espacial nas primeiras $28 \mathrm{~h}$. A partir de $30 \mathrm{~h}$, observou-se seletividades a $\mathrm{CH}_{4}$ semelhantes para as diversas velocidades espaciais. A exceção foi a de 0,4 (mol gás.g $\left.\mathrm{g}_{\mathrm{cat}}{ }^{-1} \cdot \mathrm{h}^{-1}\right)$, que só convergiu para valores semelhantes as demais, $10 \%$ de seletividade a $\mathrm{CH}_{4}$,após $60 \mathrm{~h}$.Na Figura 1(d), constata-se uma diminuição a seletividade a $\mathrm{CO}_{2}$ com o aumento da velocidade espacial, convergindo para valores próximos a $2 \%$ nas últimas horas.

\subsection{Estado estacionário}

Ao longo dos anos, vários pesquisadores vêm observando a influência das condições operacionais sobre a SFT. Ma et al. (2012), observaram que com a diminuição da velocidade espacial obtiveram um aumento na conversão de $\mathrm{CO}$, uma pequena variação na seletividade a $\mathrm{CH}_{4}$ e $\mathrm{C}_{5+}$, além de um aumento na seletividade a $\mathrm{CO}_{2}$. Iglesias et al. (1991), também observaram que o aumento da velocidade espacial diminuiu a conversão de $\mathrm{CO}$, além de um aumento no peso molecular médio dos produtos obtidos, indicando um aumento na seletividade a $\mathrm{C}_{5+}$ e diminuição na seletividade a $\mathrm{CH}_{4} \cdot \mathrm{Na}$ mesma reação, Everson et al. (1978), observaram uma diminuição na conversão e seletividade a $\mathrm{CH}_{4}$, aumentando a seletividade a $\mathrm{C}_{5+}$, com o aumento da velocidade espacial.

A Tabela 1 apresenta os valores médios obtidos nos estados estacionários de cada condição operacional citada na Figura 1.Observa-se as seletividades a $\mathrm{CH}_{4}$, a $\mathrm{CO}_{2}$ e a $\mathrm{C}_{5+}$ variam nas faixas de $3,7 \%$ a $55 \%, 0,7 \%$ a $14,1 \%$ e $38 \%$ a $95,1 \%$, respectivamente. A seletividade a $\mathrm{CH}_{4}$ é um parâmetro que indica a tendência em formar hidrocarbonetos leves na SFT, através da ineficiência em realizar reações de polimerização ou, segundo Yang et al. (2014), através da hidrogenólise como reação secundária. Diante disto, a $230^{\circ} \mathrm{C}$ obteve-se uma variação na seletividade a $\mathrm{CH}_{4}$ de $12,2 \%$ a $55 \%$, ocasionado pelo aumento da velocidade espacial. Este efeito é menos significativo nas demais temperaturas, apresentando uma seletividade a $\mathrm{CH}_{4}$ entre $5 \%$ a $10 \%$.

A seletividade a $\mathrm{CO}_{2}$ apresentou maior influência da velocidade espacial numa mesma faixa de temperatura. Sua maior faixa de variação foi a $230^{\circ} \mathrm{C}$ entre $4,9 \%$ a $14,1 \%$. Juntamente com o $\mathrm{CH}_{4}$, o $\mathrm{CO}_{2}$ é um produto indesejado. Ambos possuem os maiores valores percentuais de seletividade, em comparação aos hidrocarbonetos $\mathrm{C}_{2}-\mathrm{C}_{4}$ (não mostrados nesse trabalho). Portanto, possuem maior influência perante a formação de hidrocarbonetos pesados, ou seja, diminuem a seletividade a $\mathrm{C}_{5+}$. A seletividade a $\mathrm{C}_{5+}$ indica a quantidade de hidrocarbonetos com 5 ou mais carbonos em sua composição molecular, que são os produtos de maior valor agregado e desejados na SFT. Grande parte encontra-se na faixa de $85 \%$ a $93 \%$, indicando uma pequena influência da temperatura e da velocidade espacial nas condições estudadas. 


\section{9 a 22 de outubro de 2014 \\ Florianópolis/SC}

Tabela 1 - Principais produtos obtidos durante a SFT

\begin{tabular}{cccccc}
\hline \multicolumn{2}{c}{ CONDIÇÕES } & \multicolumn{4}{c}{ RESULTADOS } \\
\hline $\begin{array}{c}\text { Temperatura } \\
\left({ }^{\circ} \mathrm{C}\right)\end{array}$ & $\begin{array}{c}\text { Vel. Espacial } \\
\left(\text { mol gás.g }_{\text {cat }}^{-1} \cdot \mathrm{h}^{-1}\right)\end{array}$ & $\mathrm{CO}$ & $\mathrm{CH} \mathrm{CH}_{4}$ & $\mathrm{CO}_{2}$ & $\mathrm{C}_{5+}$ \\
\hline 230 & 0,4 & $99,8 \pm 0,1$ & $55,0 \pm 0,9$ & $14,1 \pm 0,1$ & $38,0 \pm 0,9$ \\
& 0,8 & $99,8 \pm 0,1$ & $23,7 \pm 0,6$ & $11,0 \pm 0,1$ & $72,0 \pm 0,6$ \\
& 1,5 & $88,3 \pm 0,4$ & $19,4 \pm 0,6$ & $6,7 \pm 0,2$ & $76,8 \pm 1,0$ \\
215 & 3,0 & $86,8 \pm 0,8$ & $12,2 \pm 0,4$ & $4,9 \pm 0,1$ & $85,3 \pm 0,5$ \\
& 0,4 & $99,8 \pm 0,1$ & $40,9 \pm 0,8$ & $12,6 \pm 0,1$ & $53,4 \pm 0,9$ \\
& 0,8 & $87,6 \pm 0,2$ & $9,9 \pm 0,7$ & $3,6 \pm 0,1$ & $88,2 \pm 0,7$ \\
& 1,5 & $69,5 \pm 0,2$ & $10,1 \pm 0,1$ & $1,9 \pm 0,1$ & $87,8 \pm 0,1$ \\
& 3,0 & $50,8 \pm 1,1$ & $5,8 \pm 0,4$ & $1,0 \pm 0,1$ & $93,1 \pm 0,4$ \\
& 0,4 & $99,7 \pm 0,1$ & $14,1 \pm 0,2$ & $8,7 \pm 0,1$ & $82,7 \pm 0,2$ \\
& 0,8 & $87,5 \pm 1,3$ & $6,1 \pm 0,3$ & $3,1 \pm 0,1$ & $92,4 \pm 0,4$ \\
& 1,5 & $45,0 \pm 0,6$ & $8,6 \pm 0,3$ & $1,3 \pm 0,1$ & $89,8 \pm 0,3$ \\
& 3,0 & $22,7 \pm 0,3$ & $6,5 \pm 0,4$ & $0,7 \pm 0,1$ & $92,2 \pm 0,4$ \\
& 0,4 & $88,2 \pm 0,1$ & $5,6 \pm 0,1$ & $2,7 \pm 0,1$ & $92,9 \pm 0,1$ \\
& 0,8 & $71,5 \pm 0,2$ & $3,7 \pm 0,1$ & $1,6 \pm 0,1$ & $95,1 \pm 0,1$ \\
& 1,5 & $26,5 \pm 0,3$ & $11,9 \pm 0,2$ & $0,9 \pm 0,1$ & $86,2 \pm 0,4$ \\
& 3,0 & $3,7 \pm 0,2$ & $8,1 \pm 0,2$ & $2,7 \pm 0,2$ & $90,0 \pm 0,0$ \\
\hline
\end{tabular}

A conversão de $\mathrm{CO}$ é o parâmetro que indica a quantidade de gás de síntese transformado em $\mathrm{CO}_{2}$, hidrocarbonetos leves e pesados. Ma et al. (2011) e Bukur et al. (2012), utilizaram catalisadores de $\mathrm{Co}$ (15-25\%), suportados em $\mathrm{Al}_{2} \mathrm{O}_{3}$, com ou sem promotores, e obtiveram conversões entre (1290\%) em vários reatores e em condições habituais da SFT. Nos dados da Tabela 1, observa-se que a conversão de $\mathrm{CO}$ varia na faixa de $3,7 \%$ a $99,8 \%$. Assim, os resultados obtidos neste trabalho, em termos de $\mathrm{CO}$, apresentam melhores resultados, quando comparado a literatura para condições similares. Esse fato pode ser devido a composição do gás de síntese, que devido a sua diluição, proporciona uma melhor distribuição de calor, evitando pontos quentes, podendo levar a uma maior estabilidade do catalisador.

\subsection{Velocidade espacial}

Na Figura 2observa-se uma tendência no decréscimo da conversão de CO com o aumento da velocidade espacial em cada isoterma. Esta tendência se encontra mais acentuada entre as velocidades espaciais 0,4 a 1,5 mol gás. $\mathrm{g}_{\text {cat }}{ }^{-1} \cdot \mathrm{h}^{-1}$ que entre 1,5 a $3,0 \mathrm{~mol}$ gás.g $\mathrm{g}_{\text {cat }}{ }^{-1} \cdot \mathrm{h}^{-1}$. Sabendo-se deste comportamento inversamente proporcional, entre a velocidade espacial e a conversão de CO, pode-se usar desta relação para obtenção de maiores quantidades de produtos. Porém, esta relação, torna-se menos favorável com a diminuição da velocidade espacial em todas as temperaturas estudadas, 0,4 mol gás. $\mathrm{g}_{\text {cat }}{ }^{-1} \cdot \mathrm{h}^{-1}$ e abaixo de $0,8 \mathrm{~mol}$ gás. $\mathrm{g}_{\text {cat }}{ }^{-1} \cdot \mathrm{h}^{-1}$, possibilitando conversões acima de $88 \%$.

Ainda na figura 2, observa-seque a influência da velocidade espacial sobre a conversão de CO se torna menos acentuada com o aumento da temperatura. Para isoterma de $230^{\circ}$, o efeito da 
velocidade espacial foi menos significativo, mostrando que essa variável tem influencia apenas sobre a seletividade dos produtos. Por outro lado, para as temperaturas de $215^{\circ} \mathrm{C}, 200^{\circ} \mathrm{C}$ e $185^{\circ} \mathrm{C}$, a $\mathrm{V}_{\mathrm{e}}$ mostra uma influencia abrupta sobre a conversão. Analisando-se o comportamento da conversão de CO para uma dada velocidade espacial, ainda na Figura 2, verifica-se um crescimento, da mesma, com o aumento da temperatura, mostrando um comportamento oposto ao da velocidade espacial. Neste sentido, para obter uma maior quantidade de produtos formados, pode acarretar a formação de produtos indesejáveis.

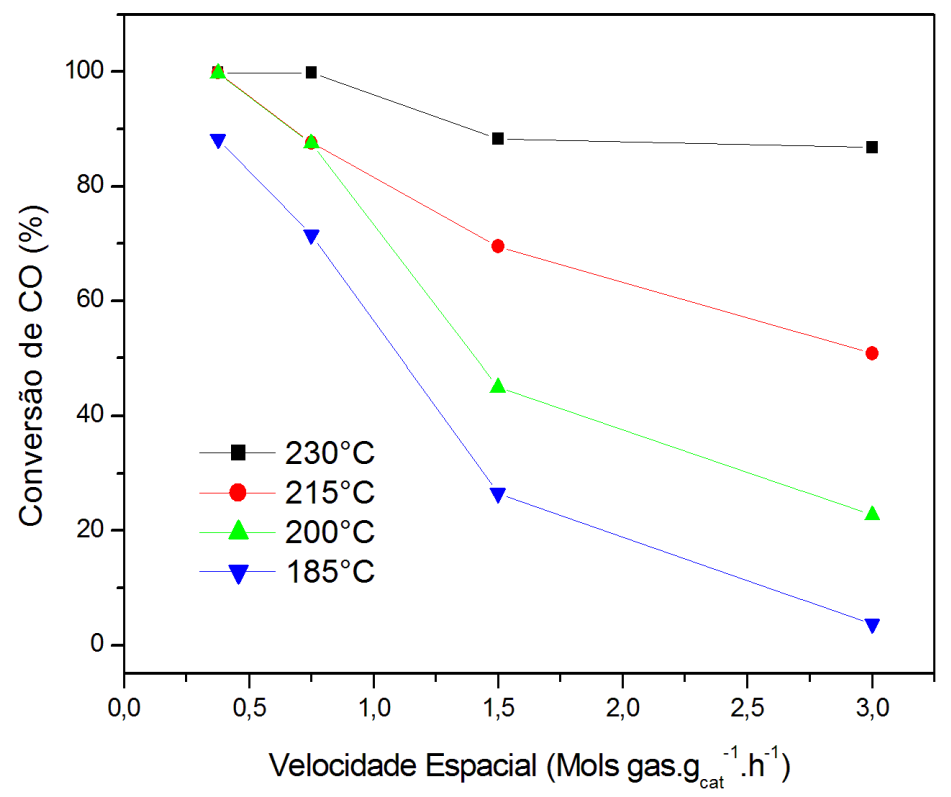

Figura 2 - Relação da conversão com a velocidade espacial nas isotermas $185^{\circ} \mathrm{C}$ a $230^{\circ} \mathrm{C}$.

\subsection{Conversão de CO}

A Figura 3 apresenta a influência da conversão de $\mathrm{CO}$ em relação a seletividade a $\mathrm{CH}_{4}$ considerando toda série de experimentos estudados neste trabalho. Como se sabe, o $\mathrm{CH} 4$ é o principal e mais fácil produto a se formar na SFT, termodinamicamente. Portanto, podemos considerá-lo como o principal indicador da HC líquidos.

Ainda, na Figura 3, de modo geral, observou-se que a seletividade a $\mathrm{CH} 4$ tende a diminuir suavemente com o aumento da conversão de $\mathrm{CO}$ até valores próximos a $60 \%$. Próximo a esta conversão de $\mathrm{CO}$, verificou-se um ponto de inflexão, que para valores maiores que ele, a seletividade a $\mathrm{CH}_{4}$ aumenta, assim, apresentando um comportamento oposto ao obtido para conversões de $\mathrm{CO}$ menores. Observando-se de forma individual o comportamento em cada temperatura, observa-se que estes pontos de inflexão variam, aumentando com o crescimento da temperatura. 


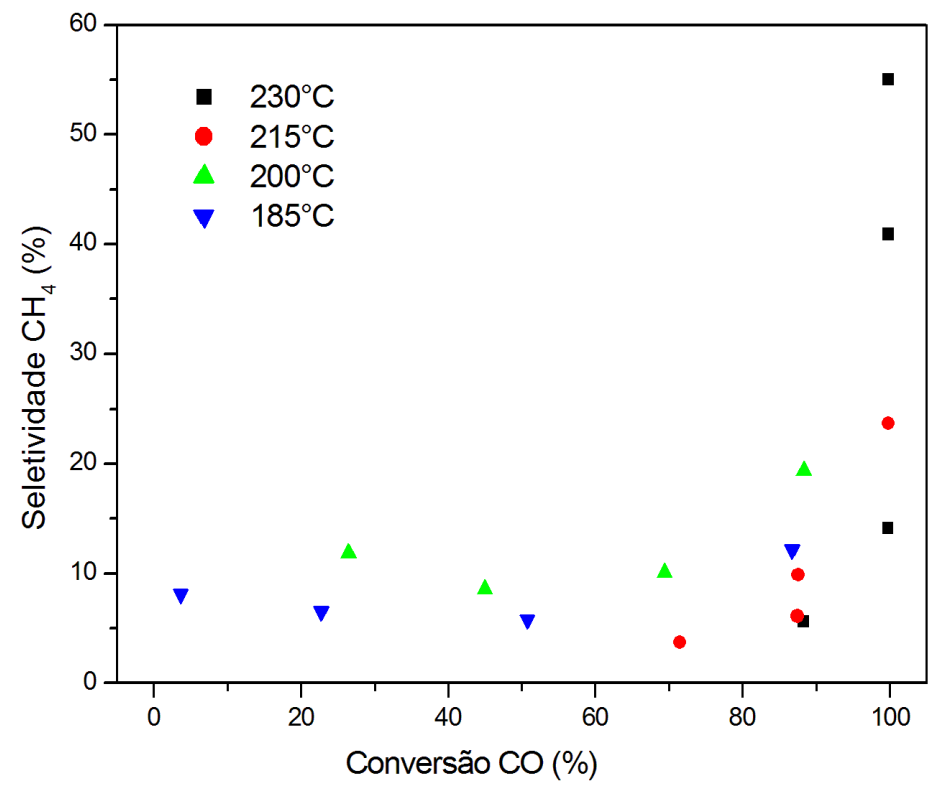

Figura 3 - Relação entre a conversão de $\mathrm{CO}$ e a seletividade a $\mathrm{CH}_{4}$.

No mesmo sentido, Yang et al. (2014), observaram uma variação na seletividade a $\mathrm{CH}_{4}$ entre 5,7 a $11 \%$ para conversões de $\mathrm{CO}$ próximas a $50 \%$, utilizando catalisadores a base de Co com diferentes promotores e suportes, corroborando com os dados obtidos na Figura 3.

Na mesma Figura, para iso-conversões acima de 80\%, observou-se que a conversão de CO sofre maior influência da temperatura. Por outro lado, a seletividade a CH4 apresenta uma maior dependência, com sua diminuição quando aumentada a velocidade espacial em iso-conversões. Podendo ser, devido ao efeito diluente, favorecendo a diminuição de pontos quentes no leito catalítico.

\section{CONCLUSÕES}

Os efeitos das variáveis de processo, temperatura e velocidade espacial, sobre indicadores da eficiência na SFT foram observados sobre um catalisador $(20 \% \mathrm{Co}-0,5 \% \mathrm{Pt} / \gamma$-Al2O3). Observou-se que a conversão de $\mathrm{CO}$ possui maior dependência da velocidade espacial para temperaturas mais baixas. A seletividade a $\mathrm{CH} 4$ mostrou um comportamento inversamente proporcional com a velocidade espacial e diretamente proporcional com a temperatura. A seletividade a $\mathrm{C} 5+$, na maioria das condições operacionais, se encontra na faixa de 85a 93\%. E principalmente, uma conversão de $\mathrm{CO}$ de aproximadamente $90 \%$, pode ser alcançada numa faixa de temperatura de 185 a $230^{\circ} \mathrm{C}$, com diferentes seletividades a $\mathrm{CH} 4$, utilizando-se o controle da velocidade espacial. Para uma mesma iso-conversão, o rendimento a hidrocarbonetos líquidos, menor \% $\mathrm{CH} 4$, é favorecido por condições temperatura e velocidades mais elevadas, onde os efeitos de diluição diminuem o comportamento exotérmico da reação. 


\section{AGRADECIMENTOS}

Os autores desse trabalho agradecem ao PRH-28, CETENE, LPC-DEQ-UFPE.

\section{REFERÊNCIAS}

BUKUR, D.B.; PAN, Z.; MA, W.; JACOBS, G.; DAVIS, B.H. Effect of CO Conversion on the Product Distribution of a $\mathrm{Co} / \mathrm{Al}_{2} \mathrm{O}_{3}$ Fischer-Tropsch Synthesis Catalyst Using a Fixed Bed Reactor. Catal.Lett., v. 142, p. 1382-1387, 2012.

DE LA OSA, A. R.; DE LUCAS, A.; SILVA, L. S.; MAROTO, J. D.; VALVERDE, J. L.; SÁNCHEZ, P. Performing the best composition of supported Co/SiC catalyst for selective FTS diesel production. Fuel, v. 95, p. 587-598, 2012.

EVERSON, R.C.; WOODBURN, E.T.; KIRK, A.R.MJ. Fischer-Tropsch reaction studies with supported ruthenium catalysts: I. Product distributions at moderate pressures and catalyst desactivation. Catal., v. 53, p. 186-197, 1978.

IGLESIA, E.; REYES, S. C.; MADON, R. J. Transport-enhanced $\alpha$-olefin readsorption pathways in Ru-catalyzed hydrocarbon synthesis.J. Catal., v. 129, p. 238-256, 1991.

JACOBS, G.; PATTERSON, P. M.; ZHANG, Y.; DAS, T.; LI, J.; DAVIS, B. H. FischerTropsch synthesis: deactivation of noble metal-promoted $\mathrm{Co} / \mathrm{Al} 2 \mathrm{O} 3$ catalysts.Appl. Catal. A: Gen., v. 233, p. 215-226, 2002.

JERMWONGRATANACHAI, T.; JACOBS, G.; MA, W.; SHAFER, W. D.; GNANAMANI, M. K; GAO, P.; KITIYANAN, B.; DAVIS, B. H.; KLETTLINGER, J. L. S.; YEN, C. H.; CRONAUER, D. C.; KROPF, A. J.; MARSHALL, C. L. Fischer-Tropsch synthesis: Comparisons between $\mathrm{Pt}$ and $\mathrm{Ag}$ promoted $\mathrm{Co} / \mathrm{Al} 2 \mathrm{O} 3$ catalysts for reducibility, local atomic structure, catalytic activity, and oxidation-reduction (OR) cycles. Appl. Catal. A: Gen., v. 464465, p. 165-180, 2013.

MA, W.; JACOBS, G.; BHATELIA,Y. J. T.; BUKUR, D.B.; KHALID, S.; DAVIS, B.H. Fischer-Tropsch Synthesis: Influence of $\mathrm{CO}$ Conversion on Selectivities, $\mathrm{H}_{2} / \mathrm{CO}$ Usage Ratios, and Catalyst Stability for a $\mathrm{Ru}$ Promoted $\mathrm{Co} / \mathrm{Al}_{2} \mathrm{O}_{3}$ Catalyst Using a Slurry Phase Reactor.Top.Catal., v. 54, p. 757-767, 2011.

MA, W.; JACOBS, G.; KEOGH, R.A.; BUKUR, D. B.; DAVIS, B. H. Fischer-Tropsch synthesis: Effect of $\mathrm{Pd}, \mathrm{Pt}, \mathrm{Re}$, and $\mathrm{Ru}$ noble metal promoters on the activity and selectivity of a 25\%Co/A12O3 catalyst. Appl. Catal. A: Gen., v. 437-438, p. 1-9, 2012

WANG, H.; ZHOU, W.; LIU, J, X.; SI, R.; SUN, G.; ZHONG, M. Q.; SU, H. Y.; ZHAO, H. B.; RODRIGUEZ, J. A.; PENNYCOOK, S. J.; IDROBO, J. C.; LI, W. X.; KOU, Y.; MA, D. Platinum-modulated cobalt nanocatalysts for low-temperature aqueous-phase Fischer-Tropsch synthesis. J Am. Chem. Soc, v. 135, p. 4149-4158, 2013.

YANG, J.;MA, W.;CHEN, D.;HOLMEN, A.; DAVIS, B. H. Fischer-Tropsch synthesis: A review of the effect of $\mathrm{CO}$ conversion on methane selectivity. Appl. Catal. A: Gen., v. 470, p. 250-260, 2014. 